\title{
EMBEDDED ACTIVE SENSORS FOR IN-SITU STRUCTURAL HEALTH MONITORING OF AGING AIRCRAFT STRUCTURES
}

\author{
Victor Giurgiutiu*, Andrei Zagrai**, JingJing Bao** \\ Department of Mechanical Engineering \\ University of South Carolina, Columbia, SC 29208 \\ Phone: 803-777-8018, FAX: 803-777-0106, E-mail: victorg@sc.edu
}

\begin{abstract}
${ }^{1}$
The aging of aerospace structures is a major current concern of civilian and military aircraft operators. Piezoelectric active sensors offer special opportunities for developing sensor arrays for in-situ health monitoring of aging aircraft fleet, because they are small, non-invasive, inexpensive and easily wired into sensor arrays. This paper presents work done on developing and utilizing piezoelectric active sensor arrays to detect near-field and far-field damage in aircraft-grade metallic plates as well as realistic builtup panels representative of aging aircraft structures. Damage due to structural cracks and corrosion was considered. For near-field damage detection, the electro-mechanical (E/M) impedance method was selected as the health-monitoring indicator. Systematic experiments conducted on statistical samples of incrementally damaged specimens were used to fully understand and calibrate the investigative method. Subsequent experiments on realistic aging aircraft panels confirmed our findings.

For far-field damage detection, guided Lamb wave ultrasonic techniques were considered. The need for high-frequency wave propagation for achieving the necessary time and space resolution in the given structural damage detection conditions was articulated and exemplified. A complete active sensor array installation and transmitter-receiver instrumentation utilizing standard laboratory equipment and $\mathrm{PC} /$ microcontroller software was constructed. Systematic experiments conducted on aircraftgrade metallic plates were used to test the pulse-echo and acousto-ultrasonic wave propagation capabilities of the system. Preliminary experiments conducted on realistic aging aircraft panels proved promising.
\end{abstract}

\section{PIEZOELECTRIC ACTIVE SENSORS}

Piezo-electric active sensors are small, non-intrusive, and inexpensive piezoelectric wafers that are intimately affixed to the structure and can actively interrogating the structure (Giurgiutiu and Zagrai, 2001a). Piezoelectric active sensors are non-resonant

*) Associate Professor, Member ASME

**) Graduate Research Assistant, Student Member ASME devices with wide band capabilities. They can be wired into sensor arrays that are connected to data concentrators and wireless communicators. Piezoelectric active sensors have captured the interest of academic and industrial community due to their low cost and small non-intrusive nature (Bartkowicz et al., 1996; Boller et al., 1999).

The general constitutive equations of linear piezo-electric material behavior, given by ANSI/IEEE Standard 176-1987, describe a tensorial relation between mechanical and electrical variables (mechanical strain, $S_{i j}$, mechanical stress, $T_{k l}$, electrical field, $E_{k}$, and electrical displacement $D_{j}$ ) in the form:

$$
\begin{aligned}
& S_{i j}=s_{i j k l}^{E} T_{k l}+d_{k i j} E_{k} \\
& D_{j}=d_{j k l} T_{k l}+\varepsilon_{j k}^{T} E_{k},
\end{aligned}
$$

where $s_{i j k l}^{E}$ is the mechanical compliance of the material measured at zero electric field $(E=0), \varepsilon_{j k}^{T}$ is the dielectric permittivity measured at zero mechanical stress $(T=0)$, and $\mathrm{d}_{k i j}$ is the piezo-electric coupling between the electrical and mechanical variables. The direct piezo-electric effect is reflected in the second equation, while the first equation refers to the converse piezo-electric effect. In this configuration, mechanical stress and strain are applied in the 1 and 2 directions, i.e. in the plane of the surface, while the electric field acts in the 3 direction, i.e., normal to the surface. Hence, the significant electromechanical couplings for this type of analysis are the 31 and 32 effects. The application of an electric field, $E_{3}$, induces surface strains, $S_{11}$ and $S_{22}$, and vice-versa. Figure 1a shows an active sensor consisting of a Lead Zirconate Titanate (PZT) piezoceramic wafer affixed to the structural surface. As the PZT sensor is activated, interaction forces and moments appear (Figure 1b):

$$
M_{a}=F_{P Z T} \frac{h}{2}, F_{P Z T}=\hat{F}_{P Z T} e^{i \omega t}, N_{a}=F_{P Z T}
$$

If the active sensor is placed on a 2-D surface, the analysis is, in principle, two-dimensional (Figure 1c). Since the electromechanical coupling constants, $d_{31}$ and $d_{32}$, have essentially same value, radial symmetry can be applied, and the analysis can be 
reduced to a one-dimensional case in the radial coordinate, $r$ (Giurgiutiu and Zagrai, 2001a).

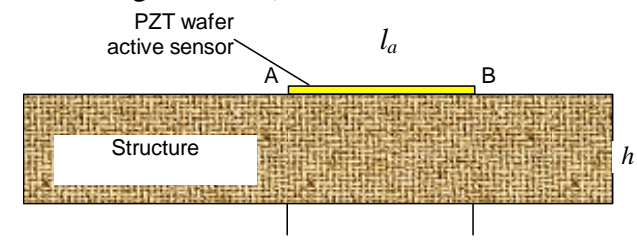

(a)

$$
x=x_{a} \quad x=x_{a}+l_{a}
$$

PZT strain and displacement, $\varepsilon_{P Z T}$ and $\mathrm{u}_{\mathrm{PZT}}$

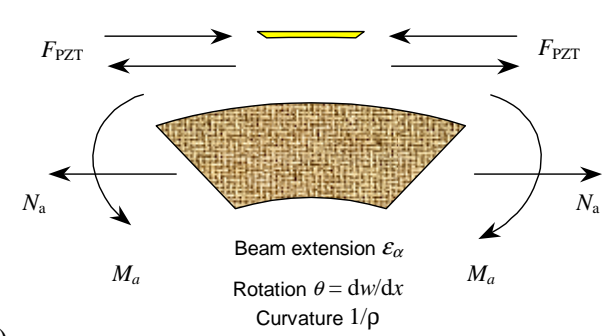

(b)

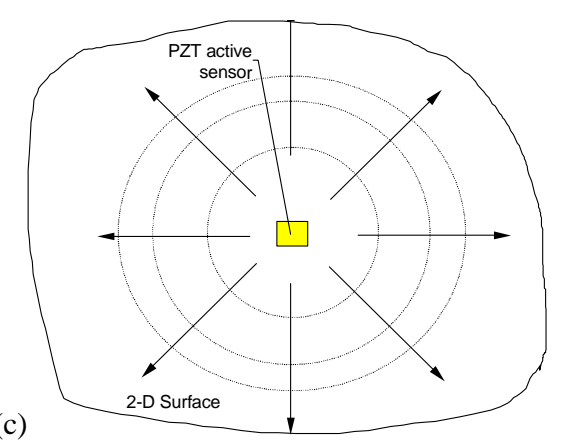

Figure 1 Piezoelectric active sensor interaction with host structure: (a) PZT wafer affixed to the host structure; (b) interaction forces and moments; (c) active-sensor elastic waves interaction on a 2-D surface (Giurgiutiu, 1999)

\section{ELECTRO-MECHANICAL (E/M) IMPEDANCE METHOD}

The impedance method is a damage detection technique complementary to the wave propagation techniques. Ultrasonic equipment manufacturers offer, as options, mechanical impedance analysis (MIA) probes and equipment (Staveley NDT Technologies, 1998). The mechanical impedance method consists of exciting vibrations of bonded plates using a specialized transducer that simultaneously measures the applied normal force and the induced velocity. Cawley (1984) extended Lange's (1978) work on the mechanical impedance method and studied the identification of local disbonds in bonded plates using a small shaker. Though phase information was not used in Cawley's analysis, present day MIA methodology uses both magnitude and phase information to detect damage.

The electro-mechanical (E/M) impedance method (Giurgiutiu and Rogers, 1997; Giurgiutiu and Zagrai, 2000) is an emerging technology that uses in-plane strain excitation at the structural surface to measure the pointwise mechanical impedance of the structure in the form of the real part of the electrical impedance at the sensor terminals. The principles of the E/M impedance technique are illustrated in Figure 2. The effect of a piezo-electric active sensor affixed to the structure is to apply a local strain parallel to the surface that creates stationary elastic waves in the structure. The drive-point impedance presented by the to the active sensor structure can be expressed as a frequency dependent quantity, $Z_{s t r}(\omega)=i \omega m_{e}(\omega)+c_{e}(\omega)-i k_{e}(\omega) / \omega$. Through the mechanical coupling between the PZT active sensor and the host structure, on one hand, and through the electromechanical transduction inside the PZT active sensor, on the other hand, the drive-point structural impedance gets directly reflected in the effective electrical impedance, $Z(\omega)$, as seen at the active sensor terminals:

$$
Z(\omega)=\frac{1}{\mathrm{i} \omega \cdot C}\left[1-\kappa_{31}^{2}\left(1-\frac{1}{\varphi \cot \varphi+r(\omega)}\right)\right]^{-1},
$$

where $C$ is the zero-load capacitance of the PZT active sensor, $\kappa_{31}$ is the electro-mechanical cross coupling coefficient of the PZT active sensor $\left(\kappa_{31}=d_{13} / \sqrt{s_{11} \bar{\varepsilon}_{33}}\right), r(\omega)$ is the impedance ratio between the pointwise structural impedance, $Z_{s t r}(\omega)$, and is the impedance of the PZT active sensor, $Z_{P Z T}$, and $\varphi=\frac{1}{2} \gamma l_{a}$, with $\gamma$ being the active sensor wave number and $l_{a}$ its linear dimension. The electro-mechanical impedance method is applied by scanning a predetermined frequency range in the high $\mathrm{kHz}$ band and recording the complex impedance spectrum. By comparing the impedance spectra taken at various times during the service life of a structure, meaningful information can be extracted pertinent to structural degradation and the appearance of incipient damage. It must be noted that the frequency range must be high enough for the signal wavelength to be significantly smaller than the defect size.

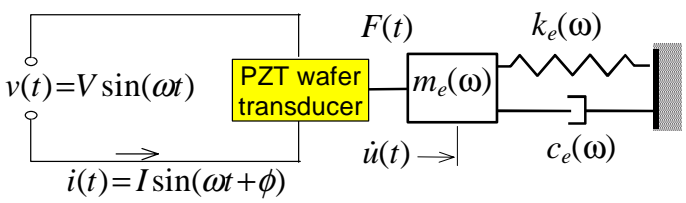

Figure 2 Electro-mechanical coupling between the PZT active sensor and the structure.

Giurgiutiu and Rogers $(1997,1998)$ presented an extensive review of the state of the art in $\mathrm{E} / \mathrm{M}$ impedance health monitoring of structures. Recent developments in this method focus on finding an effective damage metric to compare the $\mathrm{E} / \mathrm{M}$ impedance spectra of pristine and damaged structures. Lopes et al. (1999) used neural network techniques to process highfrequency E/M impedance spectra. Tseng et al. (2001) studied non-parametric damage detection metrics based on various statistical quantities.

\section{Systematic Circular-Plate Experiments}

A series of experiments on thin-gage aluminum plates were planned and conducted (Giurgiutiu and Zagrai, 2001b). Twentyfive plate specimens (100-mm diameter, $1.6-\mathrm{mm}$ thick) were constructed from aircraft-grade aluminum stock. Each plate was instrumented with one 7-mm diameter PZT active sensor placed at its center (Figure 4). A 10-mm circumferential slit EDM was used to simulate an in-service crack. The crack was placed at increasing distance from the sensor. Thus, 5 groups of five identical plates were obtained (Figure 3). E/M impedance data was taken using an HP 4194A Impedance Analyzer. During the experiments, the specimens were supported on packing foam to simulate free-free conditions. 


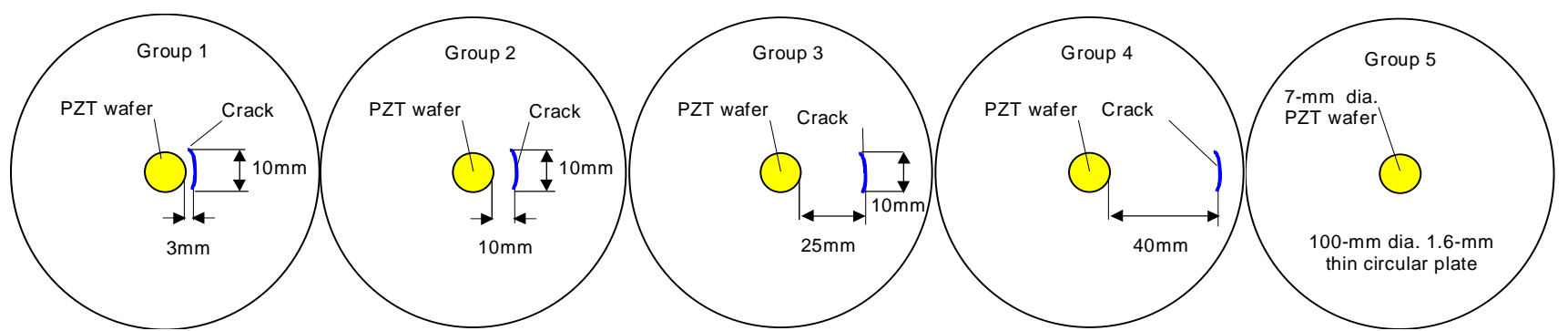

Figure 3 Systematic study of circular plates with simulated cracks (slits) at increasing distance from the E/M impedance sensor.

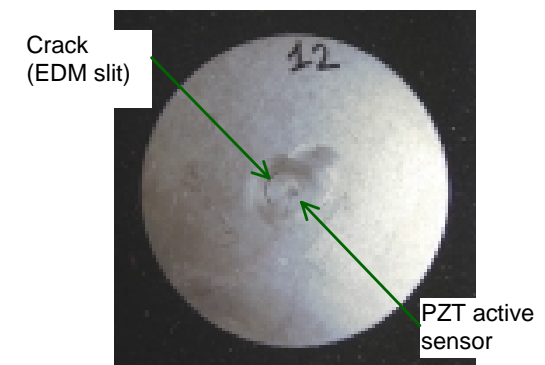

Figure 4 Photograph of circular plate specimen showing a 7-mm active sensor of the sensor and a simulated crack (EDM slit)

(a)
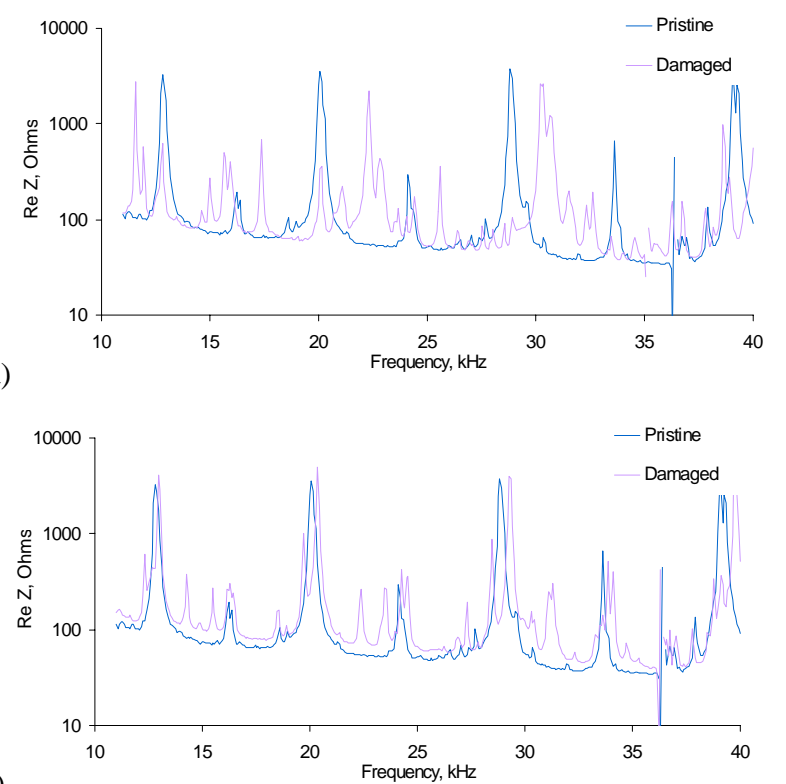

(b)

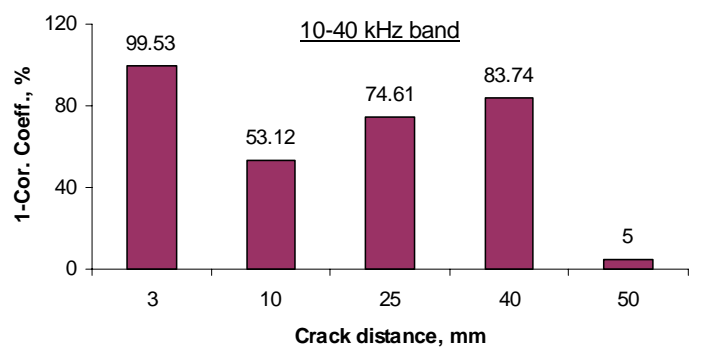

(c)

Figure 5 E/M impedance results in the $10-40 \mathrm{kHz}$ band: (a) superposed groups $1 \& 5$ spectra; (b) superposed groups 4 \& 5 spectra; (c) damage metric variation with the distance between the crack and the sensor.

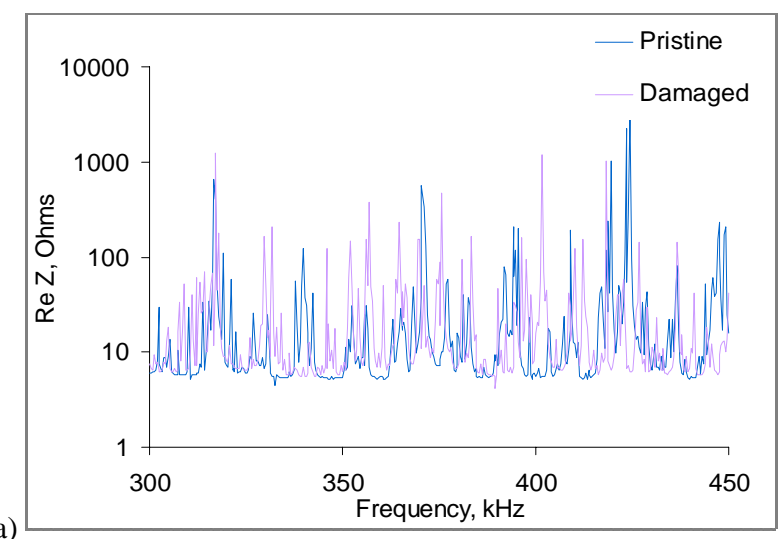

(a)
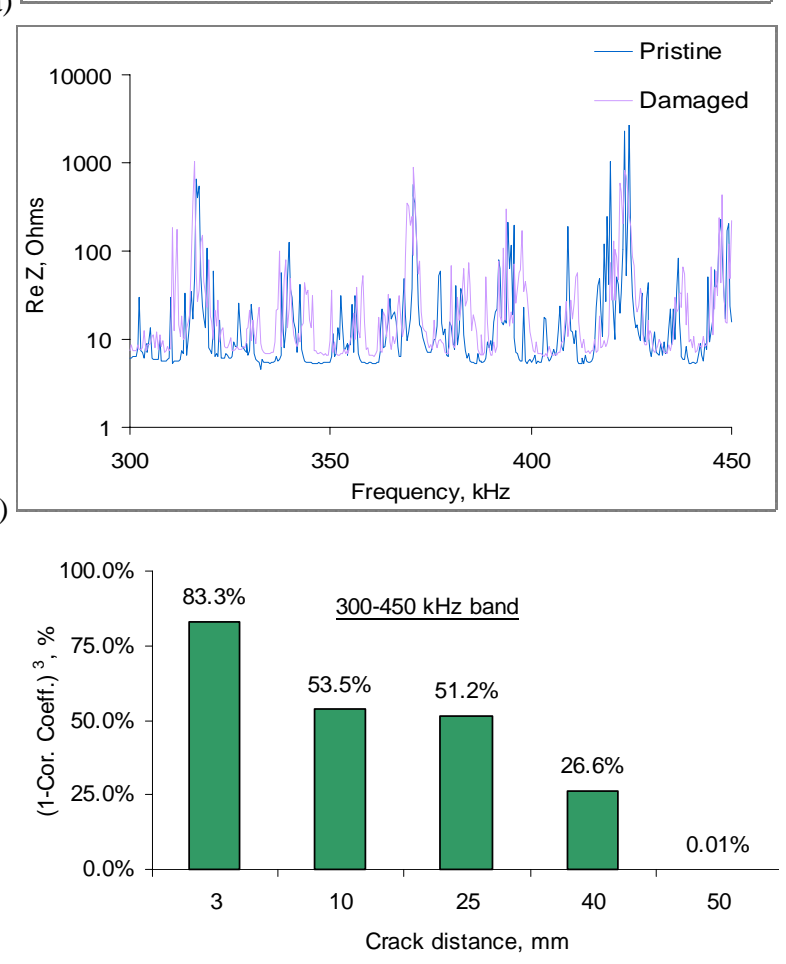

(c)

Figure 6 E/M impedance results in the $300-450 \mathrm{kHz}$ band: (a) superposed groups $1 \& 5$ spectra; (b) superposed groups 4 \& 5 spectra; (c) damage metric variation with the distance between the crack and the sensor.

The experiments were conducted over three frequency bands: 10$40 \mathrm{kHz} ; 10-150 \mathrm{kHz}$, and $300-450 \mathrm{kHz}$. The data was process by displaying the real part of the E/M impedance spectrum, and 
determining a damage metric to quantify the difference between two spectra. Several damage metrics were tried: root mean square (RMS) deviation; mean absolute percentage deviation; covariance change; correlation coefficient $\left(\mathrm{R}^{2}\right)$ deviation. Figure 5 shows data in the $10-40 \mathrm{kHz}$ band. The superposed spectra of groups 1 and 5specimens (extreme situations) are shown in Figure 5a, while those of groups 4 and 5 (almost similar situations) are shown in Figure 5b. Figure 5a indicates that the presence of the crack in the close proximity of the sensor drastically modifies the point-wise frequency response function, and hence the real part of the E/M impedance spectrum. Resonant frequency shifts and the appearance of new resonances are noticed. In contrast, the presence of the crack in the far field only marginally modifies the frequency spectrum (Figure $5 b$ ). Figure $5 \mathrm{c}$ presents the plot of the correlation coefficient deviation, $\left(1-R^{2}\right)$. The $\left(1-R^{2}\right)$ damage metric tends to decrease as the crack moves away from the sensor. However, in this frequency band, the decrease tendency is not uniform. When the higher frequency band $300-450 \mathrm{kHz}$ is examined, the damage metric decreases much more uniformly with crack distance (Figure 6c). We conclude that:

a. The crack presence dramatically modifies the pointwise frequency response function, and hence the real part of the E/M impedance spectrum

b. This modification decreases as the distance between the sensor and the crack increases

However, in order to obtain consistent results, the proper frequency band (usually in high $\mathrm{kHz}$ ) and the appropriate damage metric must be used. Further work is needed on systematically investigating the most appropriate damage metric to be used for successful processing of the frequency spectra.

\section{E/M Impedance Experiments on Aging Aircraft Panels}

Realistic aerospace panel specimens containing simulated crack and corrosion damage representative of aging-aircraft structures were designed and constructed at Sandia National Labs (Figure 8). These panels were instrumented with PZT active sensors and subjected to E/M impedance evaluation (Giurgiutiu et $a l ., 2000)$. The sensors were applied to the simulated aircraft panels to detect the change of E/M impedance spectrum induced by the proximity of a simulated crack. Figure 7 shows sensors installation: the sensors are placed along a line, perpendicular to a $10-\mathrm{mm}$ crack originating at a rivet hole. The sensors are $7-\mathrm{mm}$ square and are spaced at $7-\mathrm{mm}$ pitch. E/M impedance readings were take of each sensor in the $200-2600 \mathrm{kHz}$ range. Figure $9 \mathrm{a}$ shows the frequency spectrum of the E/M impedance real part. The spectrum reflects clearly defined resonances that are indicative of the coupled dynamics between the PZT sensors and the frequency-dependent pointwise structural stiffness as seen at each sensor location. The spectrum presented in Figure 9a shows high consistency. The dominant resonance peaks are consistently in the same frequency range, and the variations from sensor to sensor are consistent with the variations previously recorded in the simple plate experiments.

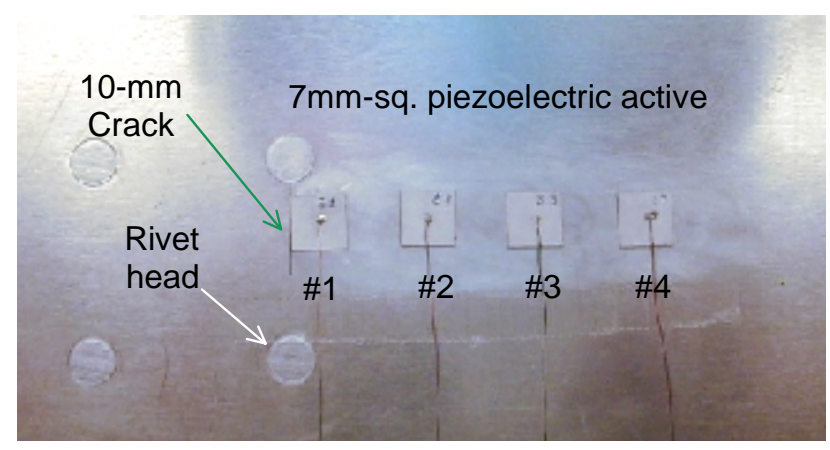

Figure 7 Piezoelectric sensors installed on the aircraft panel with aging damage simulated by a $10-\mathrm{mm}$ crack originating from a rivet.

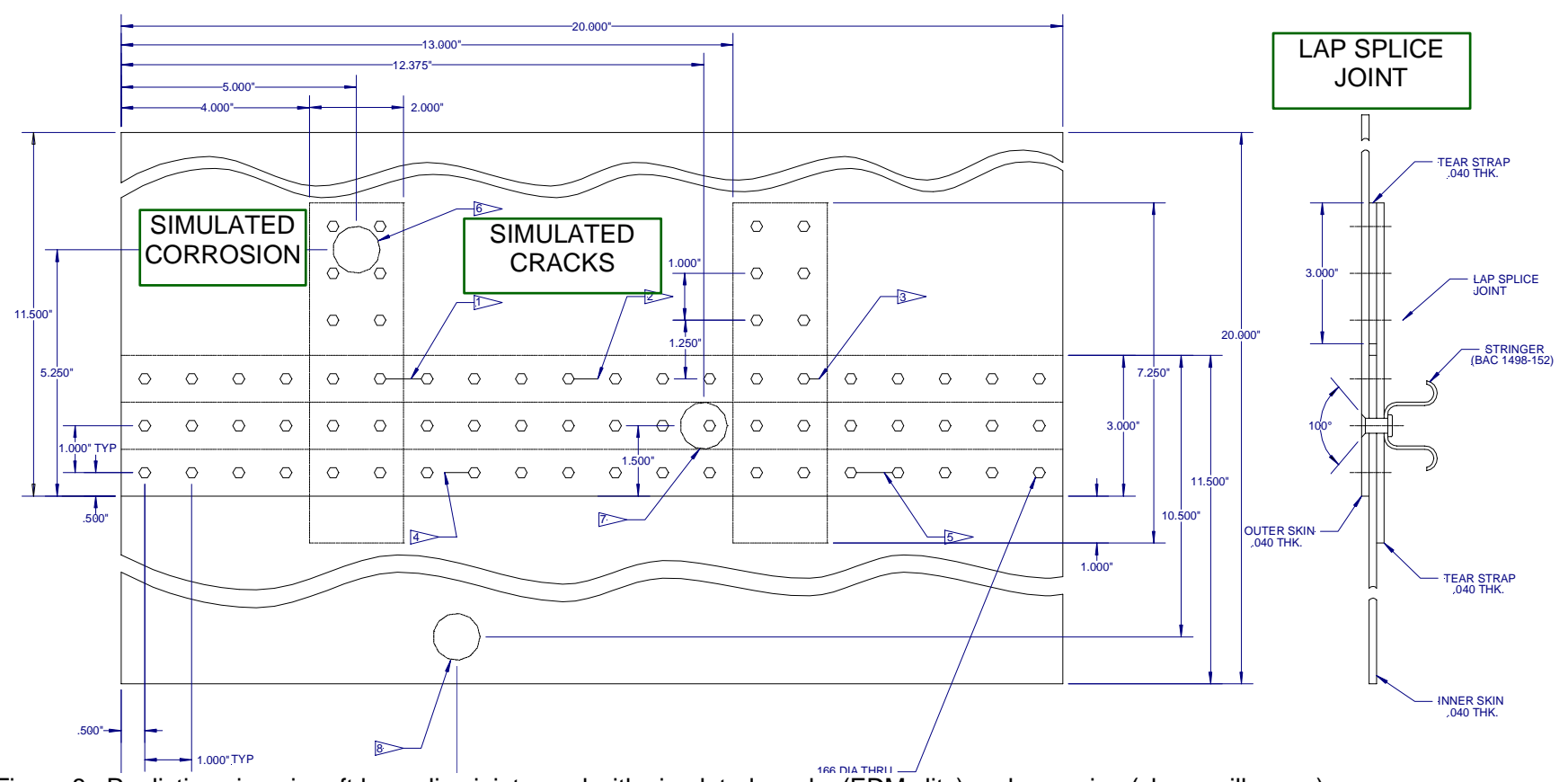

Figure 8 Realistic aging aircraft lap-splice joint panel with simulated cracks (EDM slits) and corrosion (chem-mill areas). 




(a)

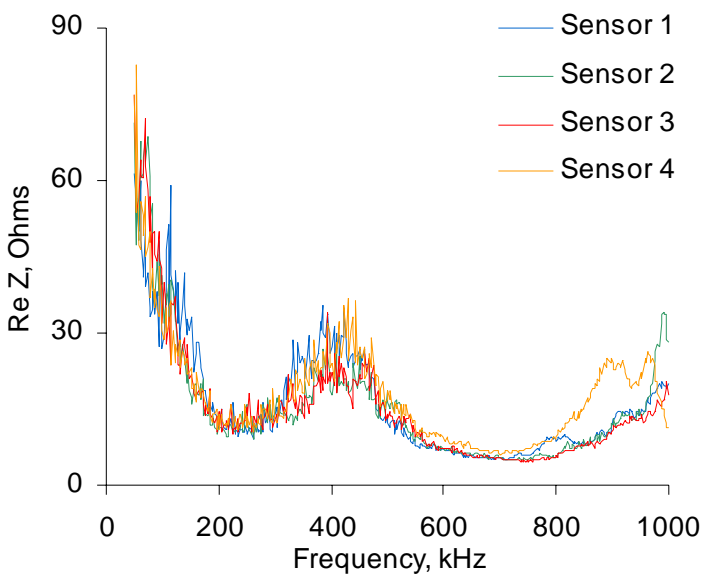

(b)

Figure 9 Real part of impedance for sensors bonded on aging aircraft structure: (a) $200-2600 \mathrm{kHz}$ range; (b) zoom into the $50-1000 \mathrm{kHz}$ range.

Examination of Figure 9a indicates that, out of the four E/M impedance spectra, that of sensor 1 (closest to the crack) has lower frequency peaks, which could be correlated to the damage presence. However, this argument is not entirely self-evident since the spectra in Figure 9a also show other sensor-to-sensor differences that are not necessarily related to the crack presence. In order to better understand these aspects, further investigations were performed at lower frequencies, in the $50-1000 \mathrm{kHz}$ range (Figure 21). In this range, we can see that the crack presence generated features in the sensor 1 spectrum that did not appear in the other sensors spectra. For example, sensor 1 presents an additional frequency peak at $114 \mathrm{kHz}$ that is not present in the other sensors. It also shows a downward shift of the $400 \mathrm{kHz}$ main peak. These features are indicative of a correlation between the particularities of sensor 1 spectrum and the fact that sensor 1 is placed closest to the crack. However, at this stage of the investigation, these correlations are not self evident, nor are they supported by theoretical analysis and predictive modeling of the structure under consideration. Further signal processing and features extraction improvements are needed to fully understand the correlation between the spectral features of the E/M impedance response and the presence of structural damage in the sensor vicinity.

\section{E/M Impedance Damage Detection Strategy}

Based on these results, we can formulate the damage detection strategy to be used with the E/M impedance method. The real part of the $\mathrm{E} / \mathrm{M}$ impedance $(\operatorname{Re} Z)$ reflects the pointwise mechanical impedance of the structure, and the E/M impedance spectrum is equivalent to the pointwise frequency response of the structure. As damage (cracks, corrosion, disbonds) develop in the structure, the pointwise impedance in the damage vicinity changes. Piezoelectric active sensors placed at critical structural locations will be able to detect these near-field changes. In addition, due to the sensing localization property of this method, far-field influences will not be registered in the E/M impedance spectrum. The integrity of the sensor itself, which may also modify the E/M impedance spectrum, is independently confirmed using the imaginary part of $\mathrm{E} / \mathrm{M}$ impedance( $\operatorname{Im} Z$ ), which is highly sensitive to sensor disbond, but much less sensitive than the real part to structural resonances (Giurgiutiu and Zagrai, 2000). To illustrate our damage detection strategy, consider an array of 4 active sensors as presented in Figure 10.

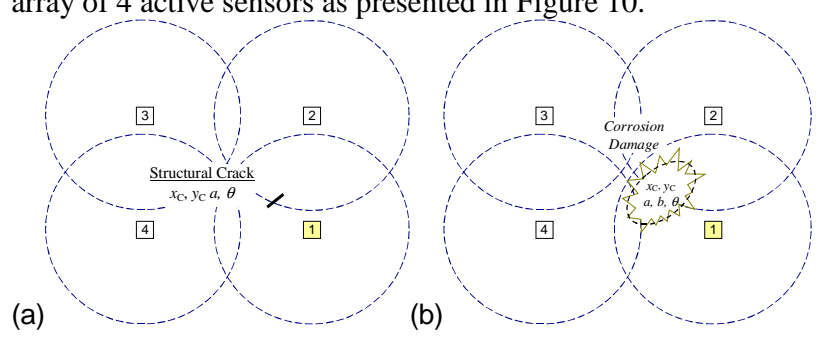

Figure 10 Damage detection strategy using an array of 4 piezoelectric active sensors and E/M impedance method: (a) detection of structural cracks; (b) detection of corrosion damage. The circles represent the sensing radius of each active sensor.

Each active sensor has its own sensing area resulting from the application of the localization concept. This sensing area is characterized by a sensing radius and the corresponding sensing circle. Inside the sensing area, the sensor detection capability decreases with the distance away from the sensor. A damage feature that is placed in the near field of the sensor is expected to create a disturbance in the sensor response that is larger than a damage feature placed in the far field. Effective area coverage is ensured when the sensing circles overlap. Structural cracks, corrosion, and disbonds/delaminations present in the sensor sensing area can be effectively detected (Figure 10). The limitations of the E/M impedance method reside in its sensing localization which diminishes its ability to detect far-field damage. For this latter case, the ultrasonic guided wave methods, discussed in the next section, may be more appropriate.

\section{GUIDED PLATE WAVES (LAMB WAVES) METHOD}

Lamb waves (a.k.a., guided plate waves) are a type of ultrasonic waves that are guided inside thin plates. For a plate having a thickness of the order of a wavelength or so, surface Rayleigh waves degenerate into Lamb waves (Viktorov, 1967). Lamb waves can propagate in a number of modes, either symmetrical or anti-symmetrical. The velocity depends on the product of 
frequency and material thickness. Investigations on Lamb and leaky Lamb waves have been carried out continuously since their discovery and researchers have done theoretical and experimental work for different purposes, ranging from seismology, to ship construction industry, to acoustic microscopy, and to nondestructive testing and acoustic sensors (Krautkramer, 1990; Rose, 1999, Lemistre et al. 1999). The Lamb wave speed is obtained by solving the Rayleigh-Lamb equation (Viktorov,

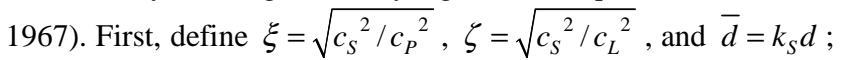
where $c_{L}$ is the Lamb wave speed, and $d$ is the half thickness of the plate. In addition, also define Lamb wave number $k_{L}=\frac{\omega}{c_{L}}$,

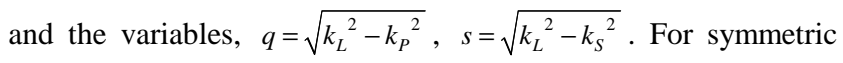
motion (Figure 11a), the Rayleigh-Lamb frequency equation can be written as

$$
\frac{\tan \left(\sqrt{1-\zeta^{2}} \bar{d}\right)}{\tan \sqrt{\xi^{2}-\zeta^{2}}}+\frac{4 \zeta^{2} \sqrt{1-\zeta^{2}} \sqrt{\xi^{2}-\zeta^{2}}}{\left(2 \zeta^{2}-1\right)^{2}}=0
$$

(a)

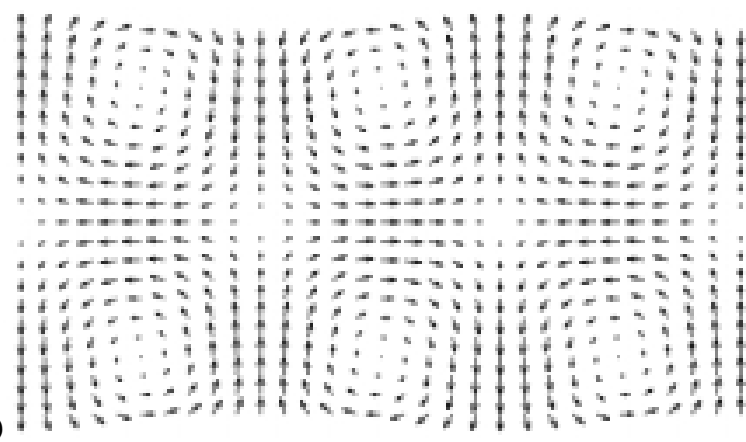

Figure 11 Simulation of Lamb wave particle motion: (a) $\mathrm{S}_{0}$ symmetric mode; (b) $A_{0}$ anti-symmetric mode

Then one can write the two components of the particle movement as:

$$
\begin{aligned}
& U(x, z, t)=\operatorname{Re}\left[A k_{L}\left(\frac{\cosh (q z)}{\sinh (q d)}-\frac{2 q s}{k_{L}^{2}+s^{2}} \frac{\cosh (s z)}{\sinh (s d)}\right) e^{i\left(k_{L} x-\omega t \frac{\pi}{2}\right)}\right] \\
& W(x, z, t)=\operatorname{Re}\left[A q\left(\frac{\sinh (q z)}{\sinh (q d)}-\frac{2 k_{L}^{2}}{k_{L}^{2}+s^{2}} \frac{\sinh (s z)}{\sinh (s d)}\right) e^{i\left(k_{L} x-\omega t\right)}\right]
\end{aligned}
$$

For anti-symmetric motion (Figure 12b), the Rayleigh-Lamb frequency equation is

$$
\frac{\tan \left(\sqrt{1-\zeta^{2}} \bar{d}\right)}{\tan \sqrt{\xi^{2}-\zeta^{2}}}+\frac{\left(2 \zeta^{2}-1\right)^{2}}{4 \zeta^{2} \sqrt{1-\zeta^{2}} \sqrt{\xi^{2}-\zeta^{2}}}=0 .
$$

The two component of the particle movement can be expressed as

$$
\begin{aligned}
& U(x, z, t)=\operatorname{Re}\left[A k_{L}\left(\frac{\cosh (q z)}{\cosh (q d)}-\frac{2 q s}{k_{L}^{2}+s^{2}} \frac{\cosh (s z)}{\cosh (s d)}\right) e^{i\left(k_{L} x-\omega t \frac{\pi}{2}\right)}\right] \\
& W(x, z, t)=\operatorname{Re}\left[A q\left(\frac{\sinh (q z)}{\cosh (q d)}-\frac{2 k_{L}^{2}}{k_{L}{ }^{2}+s^{2}} \frac{\sinh (s z)}{\cosh (s d)}\right) e^{i\left(k_{L} x-\omega t\right)}\right]
\end{aligned}
$$

Figure 12 presents the dispersive (frequency dependant) symmetric and anti-symmetric $\left(\mathrm{S}_{0}\right.$ and $\left.\mathrm{A}_{0}\right)$ Lamb wave speeds in aluminum plates. Two thickness values were considered, $2 d=1$ $\mathrm{mm}$, and $2 d=1.6 \mathrm{~mm}$. Also presented in Figure 12 is the dispersive speed for the conventional (Bernoulli-Euler) flexural waves. One notices that, at low frequencies, the conventional flexural wave and the $A_{0}$ Lamb wave speeds tend to coincide. At high frequencies, the Lamb wave speeds reach a horizontal asymptote, while the flexural waves continue to increase.

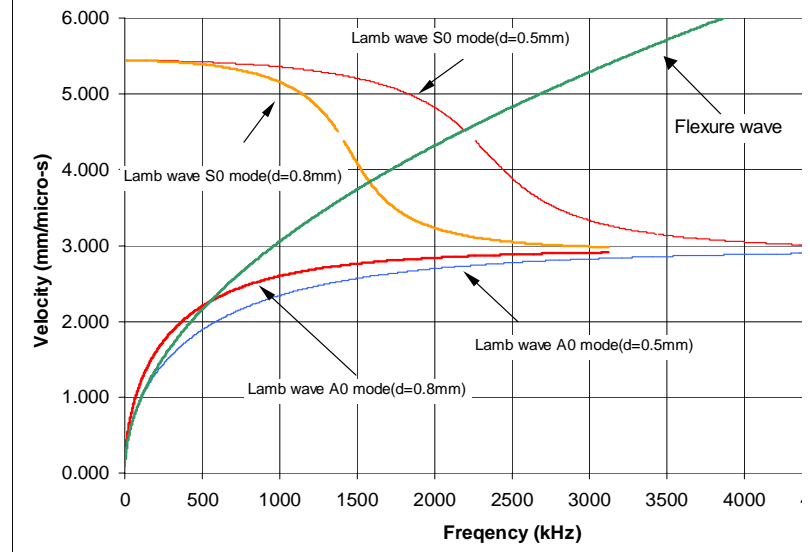

Figure 12 Wave speed dispersion curves for classical and Lamb waves in thin aluminum plates.

\section{The Need for High Frequencies Waves}

In utilizing guided waves for ultrasonic damage detection, two important aspects must be considered: (a) time resolution; and (b) defect size (detectable feature). The time resolution is especially important when using the pulse-echo method. Figure 13 shows guided Lamb waves traveling over a $800 \mathrm{~mm}$ length, first at $300 \mathrm{kHz}$ (Figure 13a), then at $20 \mathrm{kHz}$ (Figure 13b). At 300 $\mathrm{kHz}$, the time resolution is sufficient since there is a signal free zone that permits to easily establish the time of flight and detect reflected waves, if present. We also note that at this high frequency the $S_{0}$ wave gets very quickly fully established. At 20 $\mathrm{kHz}$, the time resolution is insufficient, since the signal-free zone too short and the wave is not yet fully established by the time the $800 \mathrm{~mm}$ length has been covered. At this low frequency, both $\mathrm{A}_{0}$ and $S_{0}$ waves are excited. The $S_{0}$ wave travels faster, but with a weaker amplitude. For illustration, Figure $13 \mathrm{~b}$ also presents waves detected at intermediate locations, i.e., $200 \mathrm{~mm}, 400 \mathrm{~mm}$, and $600 \mathrm{~mm}$. 
High frequency waves are also needed when detectable defect size need to be considered. Figure 14 presents the variation of wavelength with frequency for $\mathrm{S}_{0}$ and $\mathrm{A}_{0}$ Lamb waves. It is apparent that, in order to detect small size defects, high frequency waves are required. Low frequency Lamb waves can be used if the specimen length scale is large (Cawley, 1997). However, when the length scale of the specimen and/or defect size and location are small, the use high frequency Lamb waves are required.

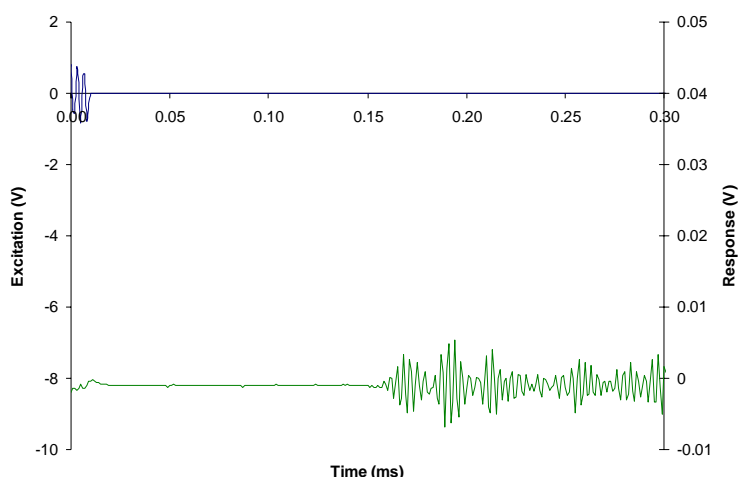

(a)

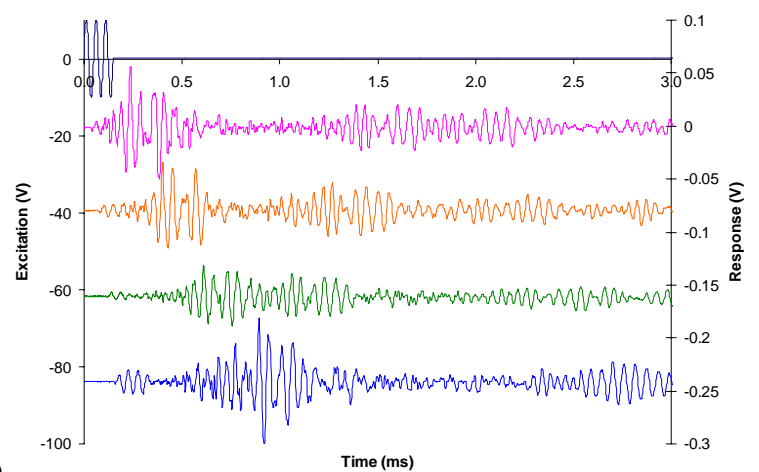

(b)

Time (ms)

Figure 13 Illustration of time resolution concept: (a) adequate time resolution at $300 \mathrm{kHz}$; (b) inadequate time resolution at $20 \mathrm{kHz}$

\section{Lamb wave Experiments on Rectangular Plates}

Previous work has been reported on using piezoelectric sensors for damage detection in composite plates (Keilers and Chang, 1995; Chang, 1998). However, since the focus of our research is on aging aircraft damage detection, our active-sensor experiments were conducted on thin metallic plates (Giurgiutiu et al., 2001). A $1.6 \mathrm{~mm}$ thick, 2024-aluminum alloy plate $(914 \mathrm{~mm} \times 504 \mathrm{~mm} \times$ $1.6 \mathrm{~mm}$ ) was instrumented with an array of eleven 7-mm x 7-mm PZT wafer active sensors. The $(x, y)$ sensor locations are given in Table 1. The sensors were connected with thin insulated wires to a 16-channels signal bus and two 8-pin connectors (Figure 15). An HP33120A arbitrary signal generator was used to generate a $300 \mathrm{kHz}$ windowed harmonic-burst excitation to active sensor \#11 with a $10 \mathrm{~Hz}$ repetition rate. Under the harmonic burst excitation, the active sensor generates a package of elastic waves that spread out into the entire plate according to a circular wave front pattern. A Tektronix TDS210 two-channel digital oscilloscope, synchronized with the signal generator, was used to collect the response signals from the active sensors. The two oscilloscope channels were digitally switched among the remaining 10 active sensors using a digitally controlled switching unit. A LabView data acquisition program was developed to control the signal switch and record the data from the digital oscilloscope. In addition, a Motorola MC68HC11 microcontroller was tested as an embedded stand-alone controlling option.

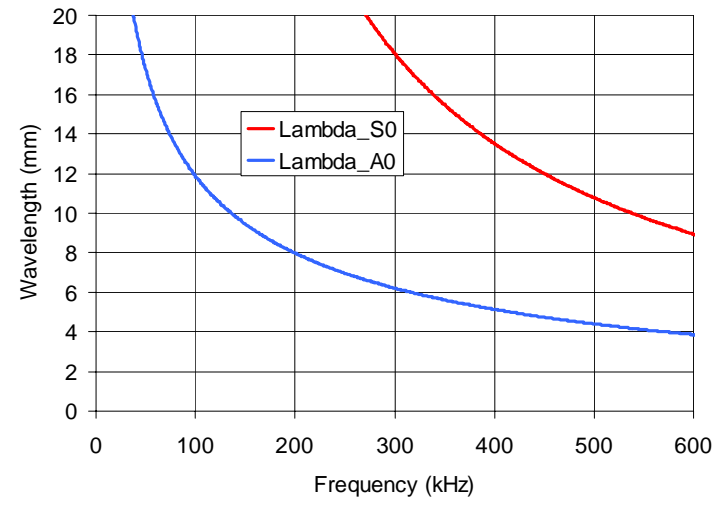

Figure 14 Wave speed dispersion curves for classical and Lamb waves in aluminum alloy medium.
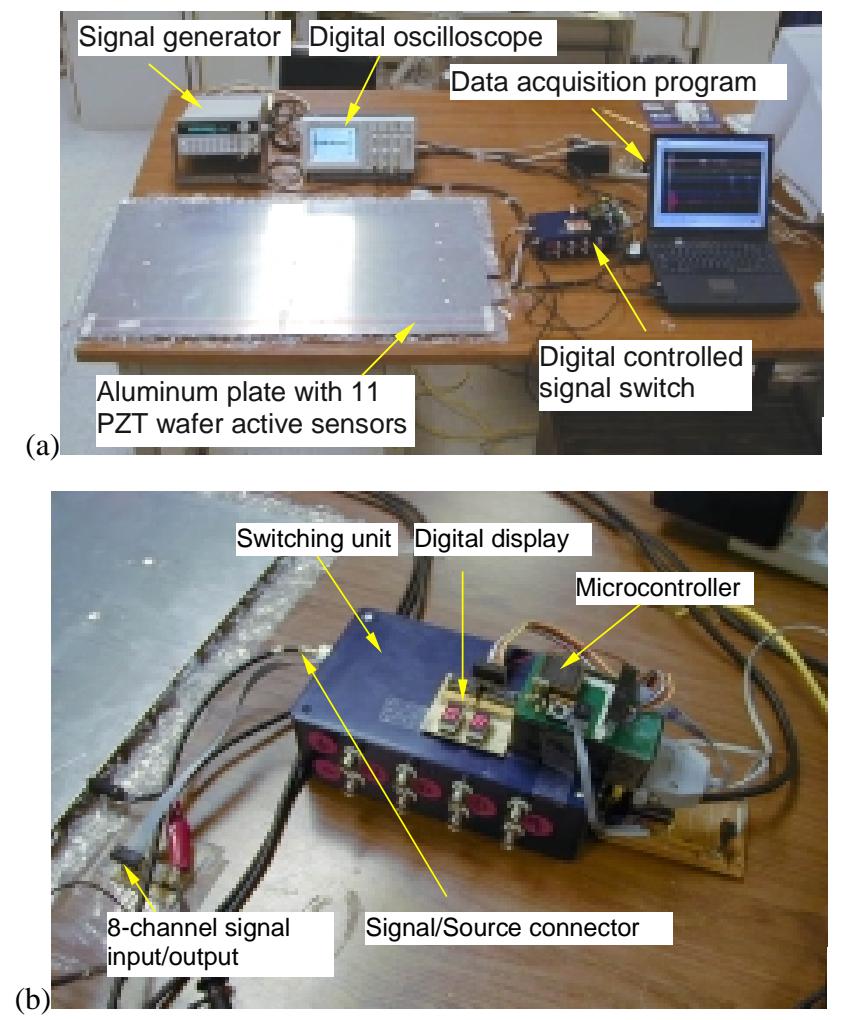

Figure 15 Experimental setup for rectangular plate wave propagation experiment: (a) overall view showing the plate, active sensors, and instrumentation; (b) detail of the microcontroller and switch box.

The results of this investigation are shown in Figure 16. The first row shows the excitation signal, and number of wave packages received in the pulse-echo mode. The wave packages are reflection from the plate edges, and their time-of-flight (TOF) position is consistent with the distance from the sensor to the respective edges. The other signal rows correspond to the other active sensors. Shown are transmitted signals. Their TOF position 
is consistent with the distance between the transmitting and receiving active sensors. The consistency of the wave patterns is remarkable. These raw signals were processed using a narrowband signal correlation algorithm followed by an envelope detection method. As a result, the exact TOF for each wave package could be precisely identified.

Table 1

\begin{tabular}{|l|l|l|}
\hline Sensor\# & $\mathrm{X}(\mathrm{mm})$ & $\mathrm{Y}(\mathrm{mm})$ \\
\hline 1 & 100 & 100 \\
\hline 2 & 100 & 175 \\
\hline 3 & 100 & 250 \\
\hline 4 & 100 & 325 \\
\hline 5 & 100 & 400 \\
\hline 6 & 450 & 100 \\
\hline 7 & 450 & 250 \\
\hline 8 & 450 & 400 \\
\hline 9 & 800 & 100 \\
\hline 10 & 800 & 250 \\
\hline 11 & 800 & 400 \\
\hline
\end{tabular}

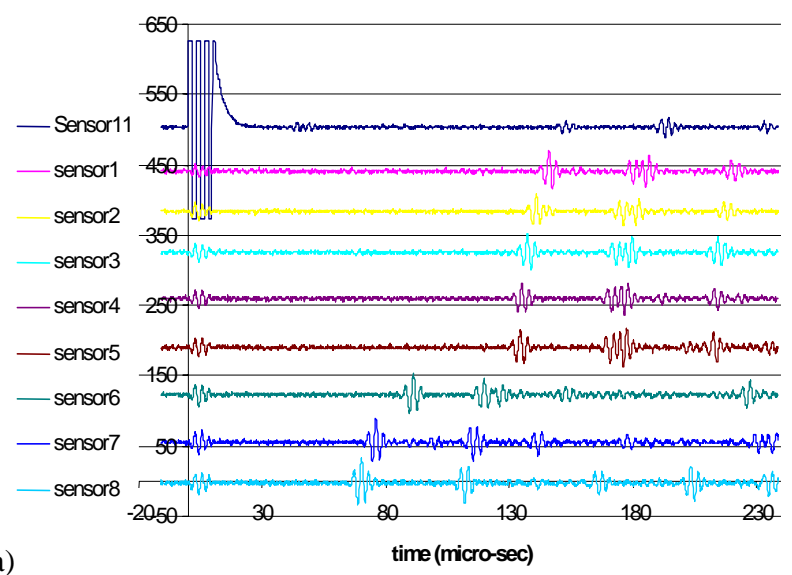

(a)

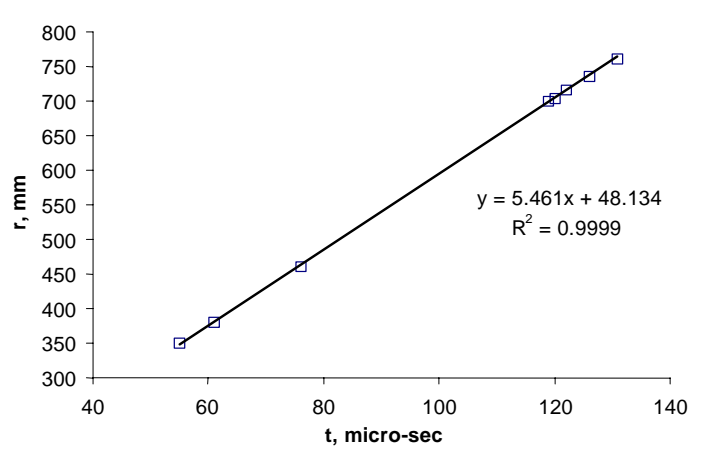

(b)

Figure 16 (a) Excitation signal and echo signals on active sensor 11 , and reception signals on the other active sensor; (b) correlation between radial distance and time of flight.

When TOF was plotted against radial distance between the receiving active sensor and the transmitting active sensor, a perfect straight line $\left(99.99 \% \mathrm{R}^{2}\right.$ correlation) was obtained (Figure 17). The slope of this straight line is the wave speed, $5.461 \mathrm{~km} / \mathrm{s}$. For the 1-6-mm aluminum alloy used in this experiment, the theoretical $\mathrm{S}_{0}$ mode speed (Figure 12) is $5.440 \mathrm{~km} / \mathrm{s}$. The speed detection accuracy $(0.3 \%)$ is remarkable. Based on the wave speed, we conclude that the first wave packets are $S_{0}$ Lamb waves. However, the subsequent wave packages are $\mathrm{A}_{0}$ mode waves. These systematic experiments have proved several important points:

c. The small non-intrusive PZT active sensors used in these experiments were capable of exciting cylindricallypropagating high-frequency Lamb waves in an aircraft-grade metallic plate.

d. The generated waves were of remarkable clarity and showed a $99.99 \%$ distance-TOF correlation that permitted accurate wave speed determination.

e. Pulse-echo methodology was successfully verified

Further to this initial experiment, we investigated the effect of simulated defects and inhomogeneities on the wave propagation pattern. The placement of a large mass, glued to the both sides of the plate at a location between the transmitter active sensor \#11 and the receptor active sensor 8 , showed masking of the first arrival wave $\left(S_{0}\right.$ mode) as expected from the acousto-ultrasonic methodology. However, the latter arrivals $\left(\mathrm{A}_{0}\right.$ mode $)$ were much less masked, which is consistent with having different particle motions in the $\mathrm{A}_{0}$ and $\mathrm{S}_{0}$ two modes.
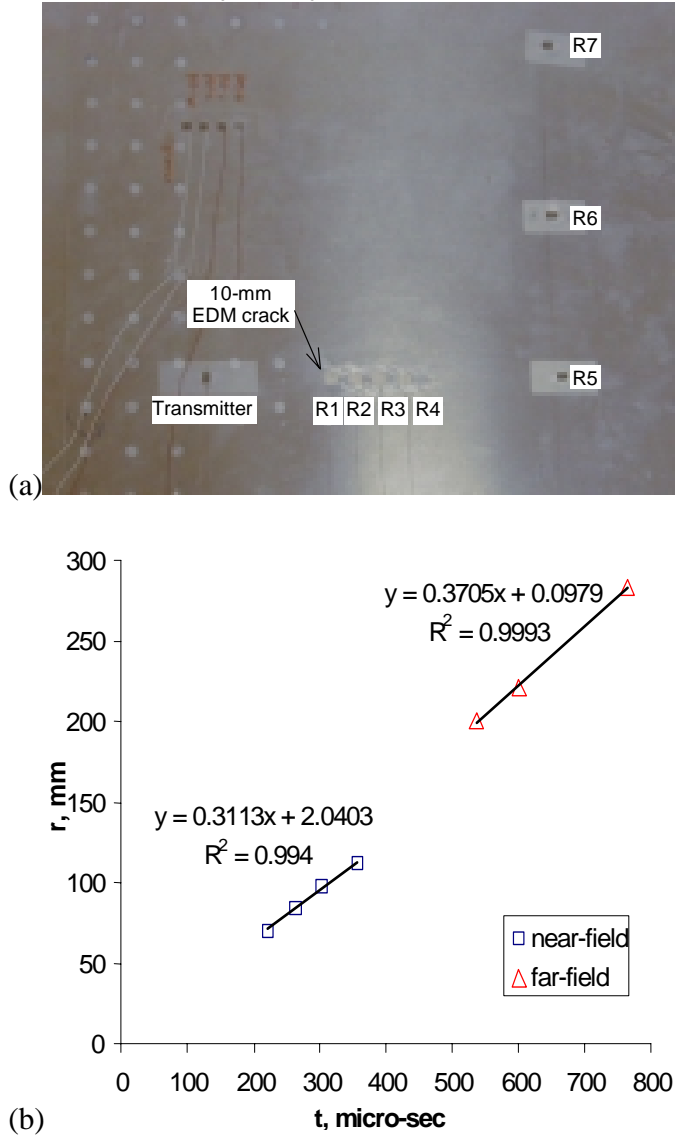

Figure 17 Lamb wave experiments on aging aircraft panel specimen: (a) sensor installation; (b) distance-TOF correlation

\section{Lamb wave Experiments on Aging aircraft Panel}

Wave propagation experiments were conducted on the aging aircraft panels with a number of PZT active sensors affixed at various locations. Wave bursts of $10 \mathrm{kHz}$ flexural $\left(\mathrm{A}_{0}\right) \mathrm{Lamb}$ waves were sent from a transmitter active sensor and collected at 
seven receptor active-sensor placed at various $x-y$ locations (Figure 17a). The correlation between distance and time of flight for the wave signals received at various sensor locations were performed (Figure 17b). Under ideal conditions, the travel distance should be directly proportional with the time of flight. The data in Figure $17 \mathrm{~b}$ is amiable to straight-line fits with a remarkable good $R^{2}$ correlation, when separated into two groups, the near-field and the far-field. The coefficient of the linear fit represents the travel speed. For the material used in these experiments (1.6- $\mathrm{mm}$ aluminum alloy sheet), the flexural wave speed at $10 \mathrm{kHz}$ is $313 \mathrm{~m} / \mathrm{s}$, i.e. $0.3130 \mathrm{~mm} / \mu \mathrm{s}$. Figure 18 shows that the near-field speed is $0.3113 \mathrm{~mm} / \mu \mathrm{s}$, while the far-field speed is $0.3705 \mathrm{~mm} / \mu \mathrm{s}$. These results are within $0.5 \%$ and $21 \%$ of theoretical, respectively. We conclude that the agreement between the theory and experiment is very good for near-field, but less good for far-field. The larger discrepancy observed in the far-field can be attributed to wave dispersion effects.

Overall, it can be concluded that the preliminary wavepropagation experiments have established that this method is a viable option for active-sensor damage detection. The limitations and challenges of the method have also been revealed.

\section{Damage detection strategy with guided lamb wave method}

The damage detection strategies using small non-intrusive active sensors and guided Lamb waves stem from the ultrasonic and acousto-ultrasonic damage-detection methodologies (Blitz et al., 1996; Duke, 1988, respectively) Consider an array of 4 active sensors as presented in Figure 18. Since piezoelectric active sensors can act as both sensors and actuators, our strategy assumes that one active sensor acts as actuator (1) while the others act as sensors (2, 3, and 4). Active sensor 1 generates elastic waves that propagate through the material and are sensed at active sensors 2, 3, and 4. To maximize the amount of data and mitigate experimental error, a round-robin process is applied, whereby active sensors 2, 3, and 4 take, in turn, the function of wave generators, with the rest of the active sensors being wave receptors. This method can be applied to detect two types of damage, cracks and corrosion.

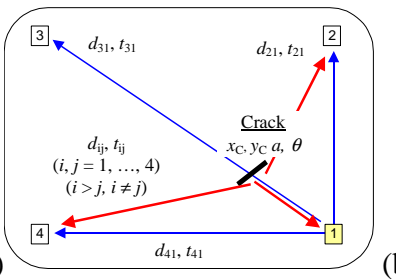

(b)

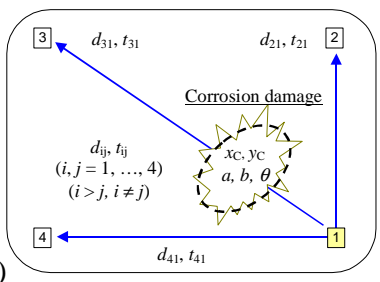

Figure 18 Damage detection strategy using an array of 4 piezoelectric active sensors and wave propagation techniques: (a) detection of structural cracks; (b) detection of corrosion damage

\section{Wave Propagation Detection of Structural Cracks}

Crack damage is characterized in terms of its location, $\left(x_{\mathrm{C}}, y_{\mathrm{C}}\right)$, and its size, $a$, and orientation $\theta$. When a crack is present in the wave path, wave deflection/scatter, reflection, and transmission at the crack is expected (Figure 18a). The proportion between deflection, reflection, and transmission will vary with damage size and orientation. In Figure 18a, active sensors 2 and 4 are shown to receive both direct and deflected waves. Active sensor 1 (the wave generator) also acts as a receptor and detects a reflected wave (echo). Active sensor 3 will receive a transmitted wave, its amplitude function of damage size. Thus, a matrix of valuable information in terms of event arrival time can be set up. Mathematical solution will yield the damage location, size and orientation $\left(x_{\mathrm{C}}, y_{\mathrm{C}}, a, \theta\right)$. In the solution algorithm, conventional linear algebra solution methods and/or neural network algorithms can be employed.

\section{Wave Propagation Detection of Corrosion Damage}

Unlike crack damage, which is 1-dimensional, corrosion damage is 2-dimensional and can cover a wide area. In Figure 18b, active sensor 1 generates elastic waves that propagate through the material and are sensed at active sensors 2, 3, and 4 . The waves will propagate through damaged material different than through the pristine material. The difference will be in wave speed and attenuation. The corrosion damage has to be characterized in terms of its location, $\left(x_{\mathrm{C}}, y_{\mathrm{C}}\right)$ and its size and orientation $(a, b, \theta$, i.e., the major and minor axes of a damage ellipse, and axis inclination). In Figure 18b, the waves received by the active sensors 2 and 4 are shown to travel through pristine material, and hence will not be affected. The waves received by active sensor 3 travel through damaged material, hence a modification in their travel time (wave speed) and attenuation is expected. (If damage is very intense, the material degradation may be very advanced, and it is possible that no waves are received at active sensor 3.) Mathematical solutions, linear algebra or neural networks, yields the damage location, size and orientation $\left(x_{\mathrm{C}}, y_{\mathrm{C}}, a, b, \theta\right)$.

\section{SUMMARY AND CONCLUSIONS}

The aging of aerospace structures is a major current concern of civilian and military aircraft operators. Piezoelectric active sensors offer special opportunities for developing sensor arrays for in-situ health monitoring of aging aircraft fleet, because they are small, non-invasive, inexpensive and easily wired into sensor arrays. This paper presents work done on developing and utilizing piezoelectric active sensor arrays to detect near-field and far-field damage in aircraft-grade metallic plates as well as realistic builtup panels representative of aging aircraft structures. Damage due to structural cracks and corrosion was considered. For near-field damage detection, the electro-mechanical (E/M) impedance method was selected as the health-monitoring indicator. Systematic experiments conducted on statistical samples of incrementally damaged specimens were used to fully understand and calibrate the investigative method. Subsequent experiments on realistic aging aircraft panels confirmed our findings. The effect of simulated damage in the form of a 10-mm crack was noticed as a left shift in the natural frequencies for the sensor closest to the crack, and the appearance of a new frequency peak at around $114 \mathrm{kHz}$. However, complete understanding of the relationship between the sensor location and the changes in the E/M spectrum has not yet been fully achieved. Further efforts to refine the automatic detection and identification of the E/M impedance spectrum features that are sensitive to the crack presence are needed.

For far-field damage detection, guided Lamb wave ultrasonic techniques were considered. The need of using high-frequency wave propagation to achieve the necessary time and space resolution in the given structural damage detection conditions was articulated and exemplified. A complete active-sensor array installation and transmitter-receiver instrumentation utilizing 
standard laboratory equipment and PC/microcontroller software was constructed and demonstrated. Systematic experiments conducted on aircraft-grade metallic plates were used to test the pulse-echo and acousto-ultrasonic wave propagation capabilities of the system. Preliminary experiments conducted on realistic aging aircraft panels proved promising. Further work on this investigative avenue requires the development of automated pulse-echo and acousto-ultrasonic data processing algorithms and calibration of the system in terms of minimal defect size and location that can be identified.

The work reported in this paper has shown that unobtrusive permanently attached PZT active sensors can be successfully used for structural health monitoring of aircraft structures in both nearfield and far-field locations. However, different methods and approaches need to be used for specific situations: $\mathrm{E} / \mathrm{M}$ impedance for the near field, and pulse-echo/acousto-ultrasonic for the far field. The advantage of the embedded active sensors method over other health monitoring options, is that both nearfield and far-field investigation can be achieved with the same active sensor array and instrumentation. When coupled with the inherent inexpensiveness and non-intrusiveness of the piezoelectric-wafer active sensors, the advantages and superiority of this emerging technology become self-evident.

\section{ACKNOWLEDGMENTS}

The financial support of Department of Energy through the Sandia National Laboratories, contract doc. \# BF 0133 is thankfully acknowledged. Sandia National Laboratories is a multi-program laboratory operated by Sandia Corporation, a Lockheed Martin Company, for the United States Department of Energy under contract DE-AC04-94AL85000.

\section{REFERENCES}

1. Bartkowicz, T. J., Kim, H. M., Zimmerman, D. C., Weaver-Smith, S. (1996) "Autonomous Structural Health Monitoring System: A Demonstration", Proceedings of the 37th AIAA/ASME/ASCE/AHS/ASC Structures, Structural Dynamics, and Materials Conference, SaltLake City, UT, April 15-17, 1996

2. Blitz, Jack; Simpson, Geoff (1996) Ultrasonic Methods of Non-Destructive Testing, Chapman \& Hall, 1996.

3. Boller, C., Biemans, C., Staszewski, W., Worden, K., and Tomlinson, G. (1999) "Structural Damage Monitoring Based on an Actuator-Sensor System", Proceedings of SPIE Smart Structures and Integrated Systems Conference, Newport CA. March 1-4, 1999

4. Cawley, P. (1997) "Quick Inspection of Large Structures Using Low Frequency Ultrasound", Structural Health Monitoring - Current Status and Perspective, Fu-Kuo Chang (Ed.), Technomic, Inc., 1997.

5. Cawley, P. (1984) "The Impedance Method for NonDestructive Inspection", NDT International, Vol. 17, No. 2, pp. 59-65.

6. Chang, F.-K. (1998) "Manufacturing and Design of Builtin Diagnostics for Composite Structures", $52^{\text {nd }}$ Meeting of the Society for Machinery Failure Prevention Technology, Virginia Beach, VA, March 30 - April 3, 1998.

7. Duke, J. C. Jr., Acousto-Ultrasonics - Theory and Applications, Plenum Press, 1988.
8. Giurgiutiu, V., and Rogers, C. A. (1997) " ElectroMechanical (E/M) Impedance Method for Structural Health Monitoring an Non-Destructive Evaluation", Int. Workshop on Structural Health Monitoring, Stanford University, CA, Sep. 18-20, 1997, pp. 433-444

9. Giurgiutiu, V., and Rogers, C. A. (1998) "Recent Advancements in the Electro-Mechanical (E/M) Impedance Method for Structural Health Monitoring and NDE, Proceedings of the SPIE's 5th International Symposium on Smart Structures and Materials, 1-5 March 1998, Catamaran Resort Hotel, San Diego, CA, SPIE Vol. 3329, pp. 536-547

10. Giurgiutiu, V., Zagrai, A. (2001a), "Embedded SelfSensing Piezoelectric Active Sensors for On-Line Structural Identification", Submitted to: Transactions of ASME, Journal of Vibration and Acoustics, January 2001.

11. Giurgiutiu, V.; Zagrai, A. (2001b) "Electro-Mechanical Impedance Method for Crack Detection in Metallic Plates", SPIE's 8th Annual International Symposium on Smart Structures and Materials, 4-8 March 2001, Newport Beach, CA, paper \#4335-22 (in press).

12. Giurgiutiu, V.; Bao, J.; Zhao, W. (2001) "Active Sensor Wave Propagation Health Monitoring of Beam and Plate Structures", SPIE's 8th Annual International Symposium on Smart Structures and Materials, 4-8 March 2001, Newport Beach, CA, paper \#4327-32 (in press).

13. Keilers, C. H., Chang, F.-K. (1995) "Identifying Delaminations in Composite Beams Using Built-in Piezoelectrics: Part I - Experiments and Analysis; Part II An Identification Method", Journal of Intelligent Material Systems and Structures, Vol. 6, pp. 649-672, September, 1995.

14. Krautkramer, Josef; Krautkramer, Herbert (1990) Ultrasonic Testing of Materials, Springer-Verlag, 1990.

15. ANSI/IEEE Std. 176 (1987), IEEE Standard on Piezoelectricity, The Institute of Electrical and Electronics Engineers, Inc., 1987

16. Lemistre, M.; Gouyon, R.; Kaczmarek, H.; Balageas, D. (1999) "Damage Localization in Composite Plates Using Wavelet Transform Processing on Lamb Wave Signals", $2^{\text {nd }}$ International Workshop of Structural Health Monitoring, Stanford University, September 8-10, 1999, pp. 861-870.

17. Lopes Jr., V., Park, G., Cudney, H., and Inman, D., (1999) "Smart Structures Health Monitoring Using Artificial Neural Network", $2^{\text {nd }}$ International Workshop of Structural Health Monitoring, Stanford University, September 8-10, 1999, , pp. 976-985.

18. Rose, J. L. (1999) Ultrasonic Waves in Solid Media, Cambridge University Press, 1999.

19. Tseng, K. K.-H.; Soh, C. K.; Naidu, A. S. K. (2001) "Non-Parametric Damage Detection and Characterization Using Smart Piezoceramic Material" Smart Materials and Structures (in press)

20. Staveley NDT Technologies, "Sonic Bondmaster ${ }^{\mathrm{TM}}$ Product Description" Kennewick, WA 99336, 1998

21. Viktorov, I. A., (1967) Rayleigh and Lamb Waves", Plenum Press, New York, 1967. 